3. Wallerstein I. Abrir las ciencias sociales. Informe de la Comisión Gulbenkian para la reestructuración de las Ciencias Sociales. Madrid: Siglo XXI Editores; 1996.

4. Perú, Comisión de la Verdad y Reconciliación Nacional. Las organizaciones sociales: las universidades. En: Comisión de la Verdad y Reconciliación Nacional: Informe final. Lima: CVR; 2003.

Correspondencia: Fernando Ríos Correa

Dirección: Ciudad Universitaria, Universidad Nacional Mayor de San Marcos, Pabellón de Ciencias Sociales. Av. Venezuela s/n, Lima 1, Perú.

Correo electrónico: 10150046@unmsm.edu.pe

\section{ESTUDIANTES DE PREGRADO: EL FUTURO DE LA INVESTIGACIÓN}

\section{[UNDERGRADUATE STUDENTS: THE FUTURE OF RESEARCH]}

\author{
Jorge Osada 1,3,a, Paulo Ruiz-Grosso ${ }^{1,3, a}$, \\ Mariana Ramos $1,2,3 a$
}

Sr. Editor: La investigación científica y su consiguiente publicación se han visto incrementadas en los últimos años, tanto en artículos como en el número revistas científicas que los presentan (1). Este crecimiento, influenciado por la facilidad de comunicación informática, ha sido aprovechado inmensamente por diversos grupos que se han desarrollado y afianzado sus posiciones como fuentes de conocimiento y referencia científica en diversas aéreas.

En la actualidad, se puede afirmar que navegar en la vasta red informática sin encontrar referencias científicas o a sus autores es algo inverosímil. A pesar de este desarrollo, y de su gran número de beneficios y beneficiarios, su potencial todavía no es aprovechado por todos los profesionales, científicos e investigadores. Esto va de la mano con la falta de información en los beneficios que la investigación trae tanto a nivel personal como en la sociedad.

Existe una gran cantidad de ideas de investigación no realizadas así como investigaciones truncadas y artículos no publicados. La relación entre los artículos no publicados que por ejemplo se presentan en un congreso y aquellos que terminan en una publicación puede llegar a ser hasta de 4 a $1^{(2,3)}$.

\footnotetext{
1 Facultad de Medicina, Universidad Peruana Cayetano Heredia. Lima, Perú.

2 Sociedad Científica de San Fernando, Facultad de Medicina, Universidad Nacional Mayor de San Marcos. Lima, Perú.

3 Grupo de Trabajo en Salud Mental, Universidad Peruana Cayetano Heredia. Lima, Perú.

a Médico Epidemiólogo.

Recibido: 05-03-10 Aprobado: 27-03-10
}

Nos encontramos en una situación similar a la que se vivió en épocas antiguas, tenemos un amplio mundo para descubrir y desarrollar, pero el temor a lo desconocido, es decir, a la investigación y su publicación, termina por atrapar a muchos. Lamentablemente, también retiene a aquellos que son los llamados a continuar o en muchos casos iniciar esta corriente de generación de conocimientos, nos referimos a los estudiantes universitarios.

Muchos estudiantes universitarios son expuestos a la comunidad científica de manera obligatoria y poco atractiva, siendo muchas veces presionados para realizar proyectos incluso sin haber desarrollado las capacidades mínimas para hacerlos. Así se desarrollan individuos que sólo ven a la investigación como un paso enigmático y perverso para cumplir un objetivo académico inmediato y no como una fuente válida de adquirir conocimientos y promover el desarrollo científico.

Incluso si existe evidencia que los estudiantes de medicina tienen un nivel aceptable de conocimientos en cuanto al proceso de investigación en sus instituciones $(4,5)$, esto no se ve plasmado en una mayor calidad o número de publicaciones. Para aquellos interesados en realizar investigación, surgen organizaciones mantenidas por los mismos alumnos como iniciativas extracurriculares que intentan suplir el serio abandono institucional en que se encuentra la formación en investigación ${ }^{(5)}$. Aun peor, muchas universidades han eliminado la necesidad de hacer investigación en forma de tesis como requisito para graduarse de pregrado sustituyéndola por programas académicos que más parecen intentos por recaudar fondos.

Aquellos que, en un acto de valentía y cierta cínica dignidad deciden embarcarse en la aventura de "la tesis" deben afrontar un mar de trabas burocráticas y de exagerado perfeccionismo en caso de caer en manos de revisores inadecuados. Parece que no se entiende que la elaboración de una tesis es, en muchos casos, un primer salto a la piscina de investigación, un proceso de aprendizaje.

Las instituciones de investigación, en muchos casos, carecen de recursos suficientes para una adecuada interacción con los alumnos durante la formación médica, limitando el acceso de los estudiantes a los verdaderos expertos y potenciales mentores hasta una etapa muy posterior en su desarrollo. En este sentido, pensamos que mejorar la comunicación entre aquellos que realizan investigación como su principal actividad y aquellos que se encuentran en formación es un paso vital para aprovechar el conocimiento alcanzado y potenciarlo. Esto debe ir de la mano con hacer flexible la currícula en la cual se deje espacio para este tipo de crecimiento personal. 
Vemos la necesidad de estrechar lazos entre los alumnos en pregrado y las instituciones dedicadas a realizar investigación independiente de una manera formal y organizada. Se deben implementar nuevas políticas curriculares donde se fomente el desarrollo personal y científico de los estudiantes, exponerlos a ideas y métodos que estos puedan entender y aplicar; y trabajar conjuntamente con ellos, y no sobre ellos, enseñándoles a superar paso a paso cada etapa de un trabajo de investigación. Al eliminar las trabas y desigualdades y al poner a investigadores reconocidos al alcance de los alumnos se estimula el desarrollo humano, así como científico, disminuyendo las dudas y los miedos intrínsecos en este tema.

Queremos terminar agradeciendo y reconociendo el esfuerzo puesto por las organizaciones científicas estudiantiles para mantener el espíritu de búsqueda del perfeccionamiento de la investigación, y, a título personal, los autores de esta carta queremos agradecer a nuestros maestros, que en algún momento de nuestra carrera, hicieron crecer la vocación por la investigación, con frases que cambiaron nuestra vida, como un sencillo "no es tan difícil".

\section{Conflictos de Interés}

Los autores declaran no tener conflictos de interés en la publicación de este artículo.

\section{REFERENCIAS BIBLIOGRÁFICAS}

1. Bliziotis IA, Paraschakis K, Vergidis PI, Karavasiou Al, Falagas ME. Worldwide trends in quantity and quality of published articles in the field of infectious diseases. BMC Infect Dis. 2005; 5(1): e16.

2. Jasko JJ, Wood JH, Schwartz HS. Publication rates of abstracts presented at annual musculoskeletal tumor society meetings. Clin Orthop Relat Res. 2003; (415): 98-103.

3. Montane E, Vidal X. Fate of the abstracts presented at three Spanish clinical pharmacology congresses and reasons for unpublished research. Eur $\mathrm{J}$ Clin Pharmacol. 2007; 63(2): 103-11.

4. Diaz C, Manrique LM, Galán E, Apolaya M. Conocimientos, actitudes y prácticas en investigación de los estudiantes de pregrado de facultades de medicina del Perú. Acta Med Peru. 2008; 25(1): 9-15.

5. Ramos-Rodriguez MI, Sotomayor R. Realizar o no una tesis: razones de estudiantes de medicina de una universidad pública y factores asociados. Rev Peru Med Exp Salud Publica. 2008; 25(3): 322-24.
Correspondencia: Jorge Osada Liy

Dirección: Av. Colonial 2619 Departamento D. Lima 1. Teléfono: (511) 9988-74202.

Correo electrónico: jorge.osada@mhperu.org

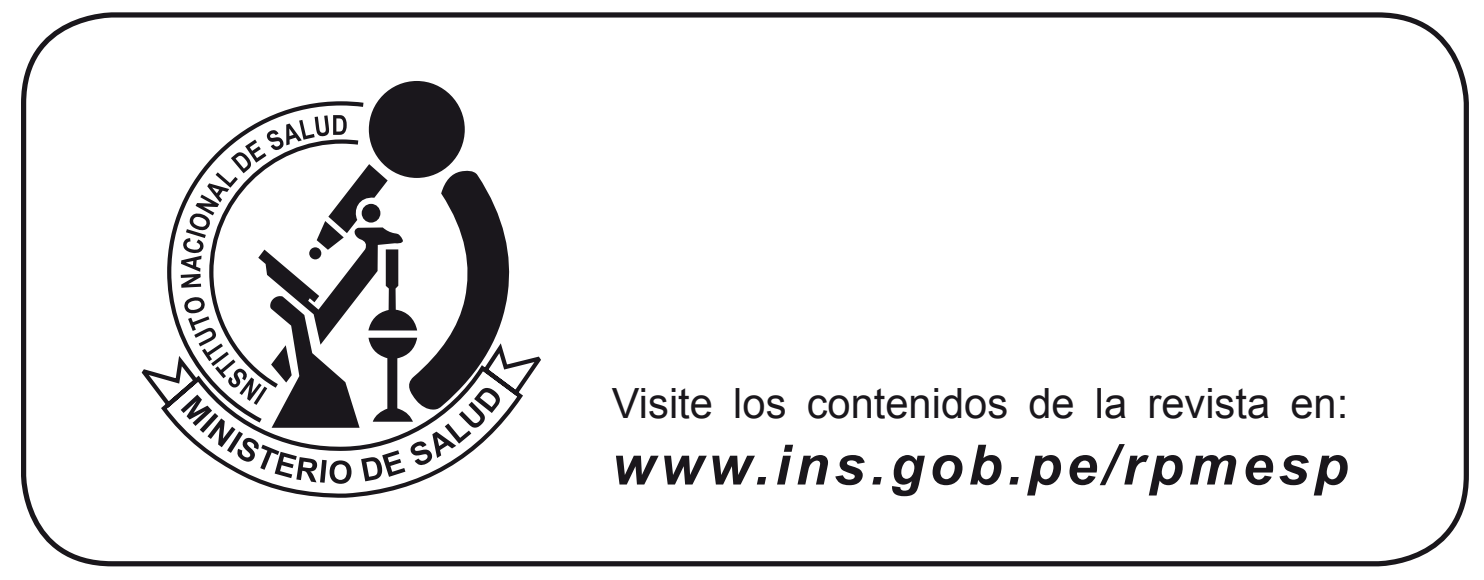

